\section{POLLUTION}

\section{Canadian Conference}

Almost 1,000 delegates attended the first International Symposium on Identification and Measurement of Environmental Pollutants (ISIMEP) held in Ottawa on June 14-17. The Conference provided a forum for scientists to report on the status of the most fundamental aspect of pollution, namely, that of delineating the problem by identifying and determining the offending substance. Thus the symposium was almost entirely devoted to chemical pollution but the potential contributions of noise and microwave radiation were briefly discussed.

Dr M. W. Holdgate of the UK Department of the Environment, in discussing the need for environmental monitoring, emphasized that effective and economic solutions to pollution problems depended on analytical assessments of the magnitude of the problem. It also had to be realized that local, national or international pollution problems demanded different approaches from the surveillance and monitoring standpoints. Dr W. Foster (US Environmental Pollution Agency) presented the broad forward requirements for combating the extensive pollution and waste problems in the United States, whereas Mr Jack Davis, the recently appointed Canadian Minister for the Environment, outlined his policy in relation to pollution problems in Canada. $\mathrm{Mr}$ Davis enunciated a basic abatement principle of "make the polluter pay", and one sensed that he would encounter some rough industrial water in pursuit of this basically laudable goal.

Drs R. A. Chapman and E. Somers (Food and Drug Directorate, Ottawa) in the session on food contamination noted that human food is the end product of a long chain of preparation and processing operations in which there are many opportunities for chemical contamination. Their review highlighted the need for methods, often sophisticated, to maintain a satisfactory level of monitoring. The need for highly sensitive and selective analytical methods, to determine extremely low concentrations of carcinogenic $\mathrm{N}$-nitrosamines in foods, was discussed by Dr J. R. Foreman (Laboratory of the Government Chemist, London). Methods based on gas chromatography with nitrogen-selective detectors coupled with high and low resolution mass spectrometry can yield detection limits of a few parts in $10^{9}$ provided suitable extraction, purification and concentration methods are available. Dr E. Somers considered the problem of heavy metal contamination of food. From analytical data, daily intake levels and existing toxicological evidence, it is concluded that mercury, cadmium and lead con- stitute the principal human health hazards. The particular problem of determining mercury, both in inorganic and organic forms, was detailed by $\mathrm{Dr} \mathrm{S}$. Nishi (Government Chemical Industrial Research Institute, Tokyo) and Dr J. F. Uthe (Fisheries Research Board of Canada, Winnipeg). A result of the work carried out both before and after the recent problem with tinned tuna fish has meant that there is now international agreement on the validity of available methods for mercury determination. Several papers showed the progress in techniques for determining pesticide residues in foods. $\mathrm{Dr} M$. Beroza and colleagues of the US Department of Agriculture, Beltsville, summarized their studies on thermionic and flame photometric detectors for determining phosphorus - containing pesticides and sampling, extraction, clean-up, determination and confirmation requirements for developing multipesticide residue methods were reviewed by Drs McCully and McLean (Food and Drug Directorate, Ottawa). Dr A. V. Holden (Freshwater Fisheries Laboratory, Pitlochry), reviewing the status of methods for determining organochlorine residues in fish, was particularly concerned with the limitations and sources of error in the methods and also the problems associated with less common chlorinated residues.

The chemical complexities of the formation of trace level carcinogenic pollutants were emphasized by Drs Hoffman and Wynder (American Health Foundation, New York) and the photochemical reactions involved in smog formation by Dr R. J. Cvetanovic (National Research Council, Ottawa). The problem of establishing primary gaseous standards at low concentrations was considered by Dr B. E. Saltzman (University of Cincinatti, Ohio) who reported that permeation tubes with lifetimes of over a year could now be prepared. The sensitivity of lichens to atmospheric pollution and their utility for detection of local pollution were reviewed by Dr Brodo (Museum of Natural Sciences, Ottawa) and Drs Schonbeck and van Haut (Landesanstalt für Immusions und Bodenneutzung. schutz des Landes, Essen).

\title{
Excess Cosmic X-rays May Come from Carbon
}

IN addition to the increasing tally of discrete X-ray sources in the sky-the $\mathrm{X}$-ray stars, galaxies, quasars, and so on-astronomers know that there is a diffuse background of $\mathrm{X}$-radiation against which the discrete sources are seen. None of the many explanations put forward for the background are completely satisfactory, most of them invoking the integrated $\mathrm{X}$-ray emission from one kind or another of extragalactic object-indeed, there seems little to choose between several proposed mechanisms. A problem which may in the end throw further light on this question, however, has arisen in the past year or two; it has been found that at energies around 0.25 to $5 \mathrm{keV}$ the background flux is greater than would be expected from measurements at higher energies. Measurements of cosmic X-rays of energies less than $1 \mathrm{keV}$ are, of course, in their infancy, most measurements during the past decade being at higher energies, and little is known, for example, about how the intensity of the excess radiation varies across the sky. Nevertheless, S. D. Verma of the Louisiana State University has been inspired to draw up an explanation in which the excess of soft $\mathrm{X}$-rays is considered due to line emission from atoms in interstellar space (see next Monday's Nature Physical Science). The hypothesis will take its place with other speculation about the origin of this radiation, and will be tested when more detailed data are available.
In brief, Verma writes that interactions between cosmic ray particles and atoms in interstellar-or intergalactic-space will give rise to line emissions which could be the explanation of the soft X-ray excess. Carbon atoms are taken as models, giving rise to the characteristic $\mathrm{K} \alpha$ line at about $0.25 \mathrm{keV}$, although there seems no reason why other elements should not be considered as well, so long as account is taken of their relative abundances.

It remains to be seen whether the improved measurements that will no doubt be available soon will show the excess to be correlated with the expected distribution of carbon atoms in the galaxy, and whether a line structure can be extracted from the spectrum. If not there are other explanations to be considered, and at this stage many astronomers will still need convincing that the excess does not have a more mundane solution in terms of radiations from the Sun which have somehow found their way into the soft X-ray detectors.

Indeed, it is probably still too early also to rule out the possibility that the excess comes from unresolved extragalactic sources, although measurements reported recently by $\mathrm{A}$. N. Bunner and colleagues (Astrophys. $J$. Lett., 167, L3 ; 1971) appear to argue against an origin outside the galaxy. Once again a detailed map of the variation of the strength of the excess with galactic latitude should clinch this ques. tion. 